\title{
Effects of erythropoietin on tooth extraction socket healing in a mouse model of periodontitis
}

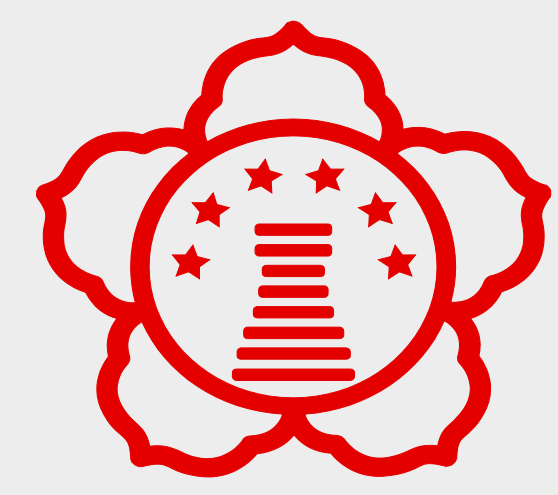

\section{Abstract}

\section{Background}

Periodontitis is defined inflammation disease of tooth-supported tissues. If periodontitis is progressive, tooth loss is induced. It may happen the dimensional change after tooth extraction. Ridge preservation allows for maintenance of the existing tissue volumes. Erythropoietin(EPO) is hormone known to stimulate the differentiation of red blood cell and recruit mesenchymal stem cells. Because of this feature, it could be believed that EPO can induce bone formation.

\section{Aim/Hypothesis}

From the perspective that EPO affects bone healing and angiogenesis, we examined the cellular effects of EPO on human periodontal ligament (hPDL) and MC3T3-E1 cells and bonehealing capacity of tooth extraction sockets in ligature induced periodontitis mouse.

\section{Materials and methods}

In vitro, after $24 \mathrm{hr}$ and $48 \mathrm{hr}$ incubation, EPO was applied to hPDL cells and MC3T3-E1 cells to detect cell proliferation using CCK-8 assay. Also we observed the expression of ALP, bone morphogenetic protein-2 (BMP-2), BMP-4, and osteocalcin (OC) genes relating to bone formation on days $1,3,5,7$ and 14 by real-time PCR.

For in vivo, Six-week-old C57BL/6 male mice were used in the experiments (total 40 mice) Mice were randomly divided into two groups of vehicle control $(n=20)$ and experimental group $(n=20)$. In both groups, the maxillary left second molars were tied for 5 days with 5-0 black silk (SK54510, AILEE CO., LTD. Busan, Korea) to induce periodontitis under anesthesia. Then we applied $10 \mathrm{IU} / \mathrm{ml}$ of EPO on extraction sockets of periodontitis induced mice and analyzed the degree of new bone formation.

Results

Test groups of hPDL cells with EPO showed the higher proliferation of cells than control group. When concentrate of EPO was $10 \mathrm{IU} / \mathrm{ml}$, it showed the highest proliferation rate. The hPDL cells with EPO upregulated the expression of ALP, BMP-2, BMP-4 and OC genes. And MC3T3-E1 cells with EPO also upregulated the expression of ALP, BMP-2 and OC genes, but decreased the expression of BMP-4 than control group. By using optical microscope, new bone formation in extraction sockets of test group showed an increased tendency compared to control group.

\section{Conclusions}

These results suggest that EPO increases the cellular activity of hPDL cells and MC3T3-E1 cells, including their proliferation and differentiation, and may play an important role in bone healing capacity of the periodontitis-induced tooth extraction sites.

\section{Background and Aim}

Periodontitis is defined inflammation disease of tooth-supported tissues. Rehabilitating the lost $5 \mathrm{~d}$ teeth with implants will be a therapeutic goal. Bone tissue characteristics are important factors in implant treatment. After tooth extraction, it happens alveolar bone dimensional change by modeling and remodeling process of the socket. And in sockets, blood clot formation occurs and many periodontal ligament cells( $P D L)$ are found near the coagulum. It has been suggested that PDL cells have regenerative capacity and affect osteogenesis in the extraction socket. Also, osteoblasts regulate osteoclastic activity and bone metabolic balance.

Erythropoietin(EPO) is a member of hematopoietic class I cytokine superfamily. Recent studies have been reported that EPO accelerated the rate of recovery and new bone formation by increasing the numbers of blood vessels, fibers, and osteoblast (20). Also, EPO promotes proliferation and the osteogenesis of the MSCs from PDL cells of the healthy and periodontitis patients.

We evaluated the cellular effects of EPO on PDL cells and MC3TC-E1 cells and bone-healing capacity of tooth extraction sockets in ligature-induced periodontitis mouse.

\section{Results}

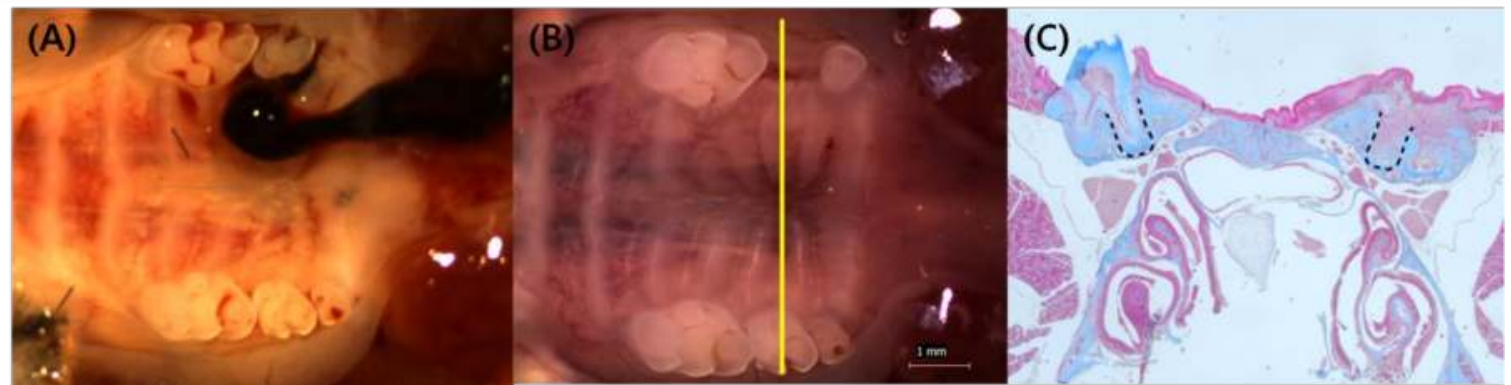

Figure 1. Induction of periodontitis on left maxillary second molar of mice and after 5 days of applying EPO $(10 \mathrm{lU} / \mathrm{ml})$ in the fresh extraction socket $(A)$, the harvested maxilla was dissected parallel to palata rugae (B). And both right and left maxillary second molars were seen on the same slide to identify the location of extraction socket (C)

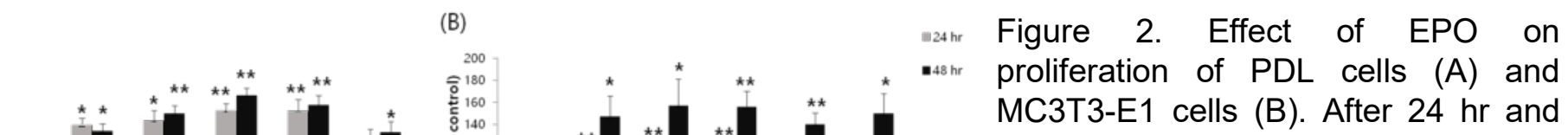 MC3T3-E1 cells (B). After $24 \mathrm{hr}$ and
$48 \mathrm{hr}$ incubation, cell proliferation assay were conducted using the Cell Counting Kit-8 (CCK-8).

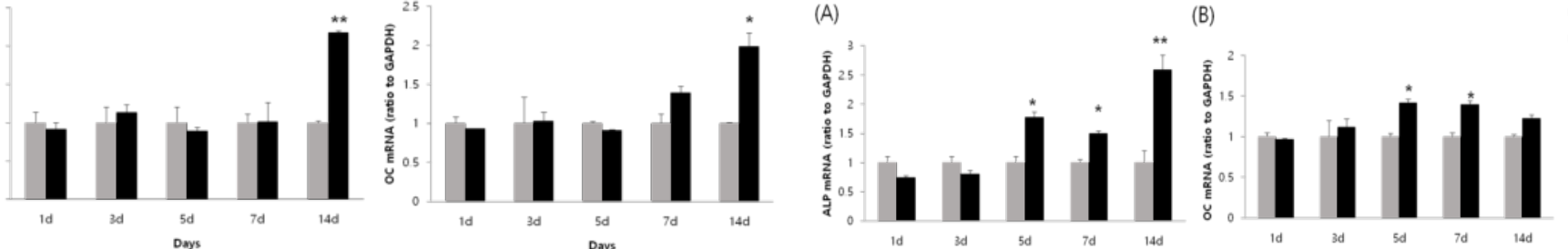

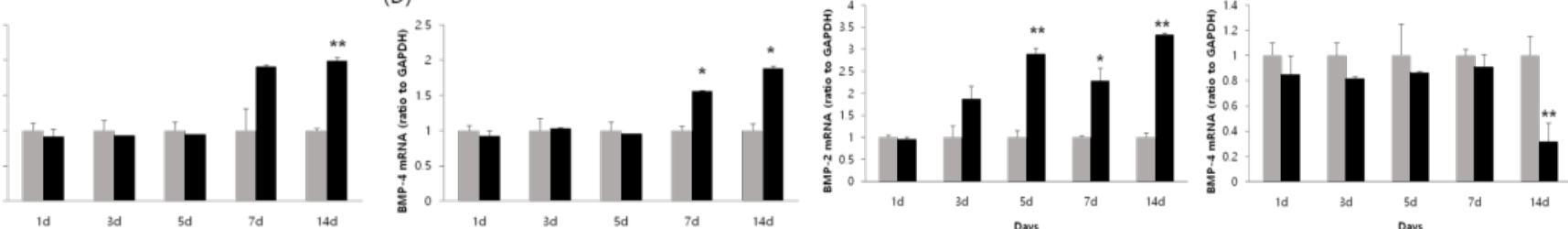

Figure 3. Effect of EPO on osteogenic gene markers (A-D) of the PDL cells. (Left)

Figure 4. Effect of EPO on osteogenic gene markers (A-D) of the MC3T3-E1 cells. (Right)
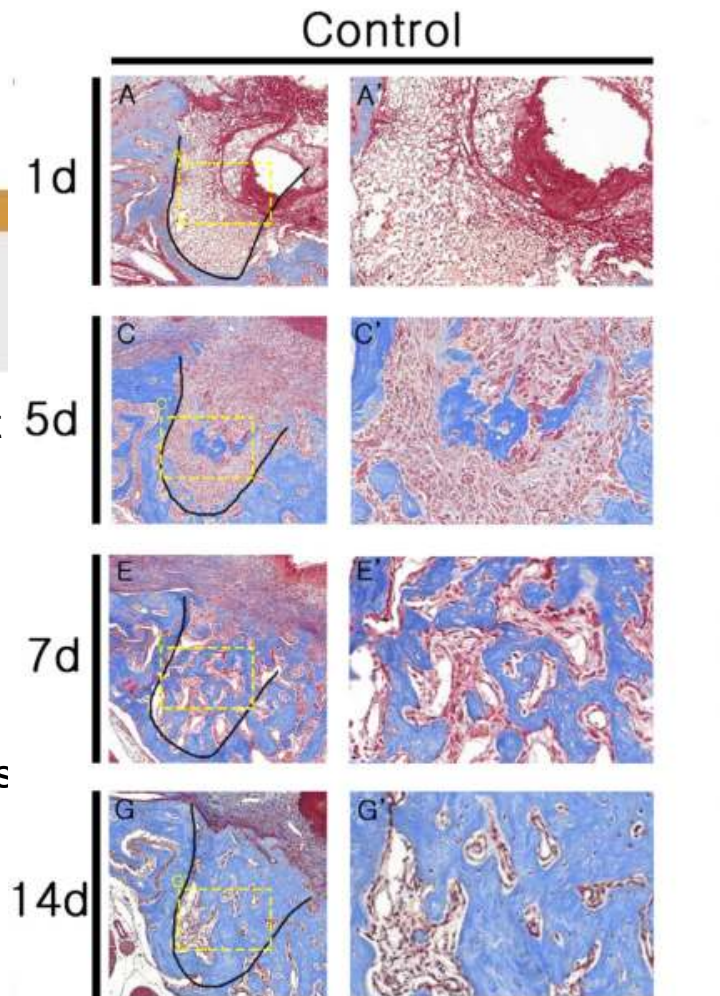

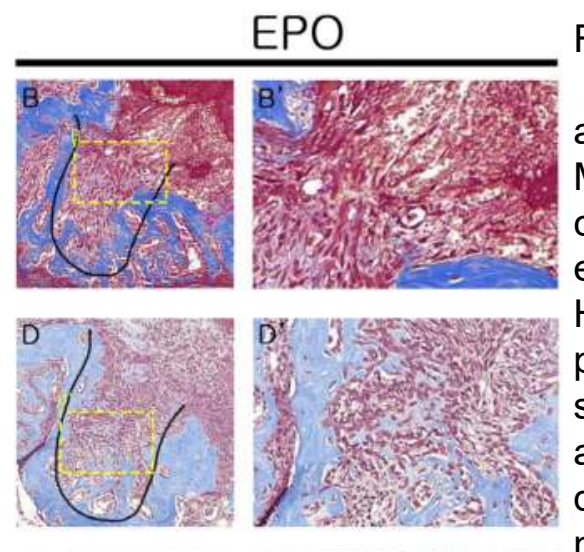

Figure 5.

Representative photomicrographs of alveolar tissue sections stained with Masson's trichrome (MTC) in the control group (A, C, E, G) and erythropoietin (EPO) group (B, D, F. H) at $1,5,7$, and 14 days. At day 1 ost treatment the coagulum filled the of Iveolar bone in both groups (A, B). At day 5 post treatment, early signs of new bone formation were observed at the PDL of the margin and the bottom of socket in the experimental group $\left(D, \quad D^{\prime}\right)$. It was observed that osteoblast was present around the blood vessels. At day 7 post treatment, the experimental group showed finger-like projection pattern of the woven bone more than the control group $\left(E, E^{\prime}, F\right.$ and $\left.F^{\prime}\right)$. At day 14 post treatment, both group showed matured bone $\left(G, G^{\prime}\right)$.

\section{Methods and Materials}

After $24 \mathrm{hr}$ and $48 \mathrm{hr}$ incubation, EPO was applied to hPDL cells to detect cell proliferation using CCK-8 assay and alkaline phosphatase (ALP) activity. And EPO was applied to MC3T3E1 cells to detect the osteogenic effect relating to bone formation.

At day $1,3,5,7$ and 14 , real-time PCR was performed to quantitate the gene expression using a specified thermal cycler with iQ SYBR Green Supermix. These genes included ALP, BMP-2, BMP-4, OC and an endogenous control gene (glyceraldehyde-3 phosphate dehydrogenase, GADPH).

Six-week-old C57BL/6 male mice were randomly divided into two groups of vehicle control (n $=20$ ) and experimental group $(n=20)$. In both groups, the maxillary left second molars were tied for 5 days to induce periodontitis with 5-0 silk. And these teeth were extracted by surgery. In the vehicle control, Pluronic F-127 was applied on the extraction socket, while the experimental group was treated with erythropoietin $10 \mathrm{IU} / \mathrm{ml}$ and Pluronic F-127. The mice were sacrificed and dissected on day 1, 5, 7, and 14 after treatments.

\section{Conclusion}

These results suggest that EPO increases the cellular activity of hPDL cells and MC3T3-E1 cells, including their proliferation and differentiation, and may play an important role in bone healing capacity of the periodontitis-induced tooth extraction sites.

\section{References}

Wang L, Wu F, Song Y, Duan Y, Jin Z. Erythropoietin induces the osteogenesis of periodontal mesenchymal stem cells from healthy and periodontitis sources via activation of the p38 MAPK pathway. international journal of molecular medicine 2018; 41: 829-835.

2. Li C, Shi C, Kim J, et al. Erythropoietin promotes bone formation through EphrinB2/EphB4 signaling. $J$ Dent Res 2015; 94: 455-463 